\title{
Archivos

\section{Procedimiento de Cox-maze III para la fibrilación auricular. Un estudio preliminar}

\section{Ovidio A. García-Villarreal*}

\author{
Departamento de Cirugía Cardíaca, Hospital de Cardiología UMAE 34, IMSS, Monterrey, Nuevo León, México
}

Recibido el 10 de noviembre de 2015; aceptado el 23 de febrero de 2016

\section{PALABRAS CLAVE \\ Arritmia; \\ Aurícula; \\ Cox-maze; \\ Fibrilación auricular; \\ México; \\ Procedimientos \\ quirúrgicos cardíacos; \\ Válvula mitral}

\section{KEYWORDS}

Arrhythmia;

Atria;

Cox-maze;

Atrial Fibrillation;

\begin{abstract}
Resumen
Objetivo: Comparar la eficacia del procedimiento de Cox-maze III mediante corte-y-sutura con el procedimiento de Cox-maze IV aplicando radiofrecuencia intraoperatoria con una pinza bipolar.

Material: De enero del 2011 a octubre del 2014, se operaron 50 pacientes por cirugía para fibrilación auricular. Todos los pacientes fueron sometidos a cirugía valvular mitral como procedimiento predominante, $y$ secundariamente se les realizó un procedimiento quirúrgico para eliminar la fibrilación auricular. Se dividieron en 2 grupos: grupo I (Cox-maze III, corte-y-sutura) y grupo II (Cox-maze IV, radiofrecuencia bipolar). El grupo I constó de 36 pacientes, y el grupo ॥ de 14 pacientes. Todos los casos tenían fibrilación auricular de más de un año de evolución. El punto final del estudio fue la ausencia de fibrilación auricular.

Resultados: No hubo diferencia estadísticamente significativa entre las características basales ni operatorias de ambos grupos. La mortalidad operatoria fue de 2 casos para el grupo I vs. 0 en el grupo ॥ $(p=0.9)$ Se observó una fuerte tendencia para la desaparición de la fibrilación auricular favorable al grupo I ( 92 vs. $53 \%, p<0.001)$ en un seguimiento a 6 meses.

Conclusiones: El procedimiento de Cox-maze III clásico de corte-y-sutura mostró superioridad para eliminar la fibrilación auricular sobre el Cox-maze IV con pinza bipolar de radiofrecuencia en pacientes con enfermedad valvular mitral y fibrilación auricular concomitante.

๑ 2016 Instituto Nacional de Cardiología Ignacio Chávez. Publicado por Masson Doyma México S.A. Este es un artículo Open Access bajo la CC BY-NC-ND licencia (http://creativecommons. org/licencias/by-nc-nd/4.0/).
\end{abstract}

\section{Cox-maze III procedure for atrial fibrillation. A preliminary study}

\section{Abstract}

Aim: To compare the efectiveness of the cut-and-sew Cox-maze III procedure against the Coxmaze IV peocedure by means of intraoperative bipolar radiofrequency delivery clamp.

\footnotetext{
* Sierra Nayarita 143, Col. Virginia Tafich; Santa Catarina, Nuevo León. CP: 66374, Teléfono: +52 18183888917 , celular: +5218182599519.

Correo electrónico: ovidiogv@hotmail.com
} 
Mexico;

Cardiac surgical

procedures;

Mitral valve
Material: From January 2011 to October 2014, 50 patients were operated on with surgery for atrial fibrillation. All cases underwent mitral valve surgery as the first procedure, and secondarily a surgical procedure for atrial fibrillation was also performed. There were 2 groups. Group I (Cox-maze III «cut-and-sew»), and Group II (Cox-maze IV, intraoperative bipolar radiofrequency ablation). Group I was formed by 36 patients, and Group II by 14. All cases had atrial fibrillation longer than 1 year. The end-point was freedom of atrial fibrillation.

Results: There was no statistically significant difference between both groups regarding the basal and operative characteristics. Operative mortality was of 2 cases in the Group I, and no cases for Group II $(P=0.9)$. A high tendency to eliminate atrial fibrillation in favour of Group I was observed ( $92 \%$ vs $53 \%, P<.001)$ in a 6 months follow-up.

Conclusions: Classic standard Cox-maze III procedure showed superiority to eliminate atrial fibrillation over the Cox-maze IV procedure made with bipolar radiofrequency ablation clamp in patients with concomitant mitral valve disease.

( 2016 Instituto Nacional de Cardiología Ignacio Chávez. Published by Masson Doyma México S.A. This is an open access article under the CC BY-NC-ND license (http://creativecommons. org/licenses/by-nc-nd/4.0/).

\section{Introducción}

La fibrilación auricular (FA) es la arritmia más común en el mundo. En 2010 se estimó que había más de 5.2 millones de casos en EE. UU. ${ }^{1}$. La FA está asociada con un aumento en la mortalidad de 1.5-1.9 veces ${ }^{2}$. El riesgo de accidente vascular cerebral (AVC) aumenta hasta 5 veces cuando está presente la FA, y hasta 17 veces cuando es de origen valvular ${ }^{3}$. La FA causa entre el 15 y $20 \%$ de los AVC isquémicos ${ }^{4}$. La mortalidad para la asociación de AVC y FA es del $50 \%$ a un año, y del $11 \%$ a un mes $^{5}$. El área cerebral afectada por un AVC debido FA es mayor cuando se compara con otro no relacionado con FA (52 vs. $16 \mathrm{cc})^{5}$. Según datos aún no publicados por el autor, hasta el $48 \%$ de los pacientes que llegan a quirófano para ser sometidos a cirugía valvular mitral presentan FA persistente de larga evolución (más de un año). Independientemente de su origen, la sola presencia de FA puede ser motivo para anticoagulación oral a largo plazo ${ }^{6}$. Sin embargo, el riesgo de hemorragia intracraneal es hasta del $2.5 \%$ anual relacionado con el uso de anticoagulantes orales, especialmente con niveles de INR superiores al 3.07,8. Por otra parte, los nuevos anticoagulantes orales no han probado tener efectividad en los pacientes con FA de origen valvular.

El procedimiento de Cox-maze III está diseñado para tratar en forma efectiva cualquier tipo de FA, incluyendo el flutter $^{9}$. En este sentido, la efectividad del procedimiento de Cox-maze III es superior al $90 \%^{10}$. La toma de decisión en el momento quirúrgico valvular debe de ir acompañada de un enfoque dirigido a eliminar simultáneamente la FA. El resultado último que se busca con este tipo de decisiones es evitar el tratamiento con anticoagulantes orales a largo plazo. Tradicionalmente, el procedimiento de Cox-maze III estándar de corte-y-sutura clásico ha sido confinado por las guías clínicas exclusivamente para aquellos pacientes que van a ser sometidos a otro procedimiento quirúrgico cardíaco primario, que muestren recidivas después de 2 o más ablaciones con catéter, intolerancia a los antiarrítmicos, y por preferencia expresa del paciente ${ }^{11}$. Esto es debido a su gran invasividad y complejidad en ejecución. De hecho, el grado de complejidad para realizar un procedimiento de
Cox-maze III clásico estándar de corte-y-sutura es 9.5 en una escala del 1 al $10^{12}$.

No obstante, el procedimiento de Cox-maze III ofrece una excelente alternativa para aquellos casos que van a ser sometidos a cirugía valvular mitral con FA concomitante.

\section{Material y método}

Cincuenta pacientes con enfermedad valvular mitral reumática y FA fueron sometidos a cirugía valvular mitral además de un procedimiento de Cox-maze III o IV, entre enero del 2011 y octubre del 2014 en nuestra institución. La población total de pacientes estudiados se dividió en 2 grupos, dependiente del tipo de procedimiento de maze utilizado: grupo I (Cox-maze III estándar de corte-y-sutura) 36 casos, y grupo " (Cox-maze IV con aplicación intraoperatoria de radiofrecuencia bipolar) 14 casos. Las características preoperatorias basales de todos los pacientes se muestran en la tabla 1. No se encontró ninguna diferencia estadísticamente significativa entre ambos grupos. En todos los casos, la duración preoperatoria de la FA fue mayor a un año de evolución. El punto final a analizar fue la ausencia de FA después de 3 meses de la cirugía. En todos los casos se realizó un estudio Holter de ritmo cardíaco de 24 h, a los 3 y 6 meses postoperatorios.

Todos los casos se realizaron a través de esternotomía media y en derivación cardiopulmonar. En los casos del grupo I se realizó un procedimiento de Cox-maze III clásico estándar de corte-y-sutura exclusivamente, siguiendo un patrón completo y biauricular de las incisiones quirúrgicas, según ha sido descrito por Cox et al. ${ }^{9}$. En los casos del grupo ॥ se recurrió a la aplicación de energía de radiofrecuencia a través del uso de una pinza bipolar.

Los valores de $\mathrm{p}$ se calcularon utilizando la prueba $\mathrm{Z}$ para 2 proporciones de población, y la t-Student para 2 medias independientes. La prueba de Chi cuadrada se utilizó para la asociación entre 2 variables categóricas. Finalmente, se utilizó un paquete estadístico SSPS (versión 15.0; SPSS, Inc., Chicago, IL, EE. UU.). 
Tabla 1 Características preoperatorias de los pacientes

\begin{tabular}{llll}
\hline Variable & Grupo I & Grupo II & P \\
Tipo Cox-maze & III (corte-y-sutura) & IV (RF) & 0.313 \\
\hline Total pacientes & 36 & 14 & 0.365 \\
Edad (años) & $51.7 \pm 8.4$ & $56 \pm 6.5$ & 0.582 \\
Mujeres & 26 & 9 & 0.582 \\
Hombres & 10 & 5 & 0.297 \\
CF (NYHA) & $2.4 \pm 0.5$ & $2.1 \pm 0.4$ & 0 \\
Mitral & 36 & 14 & 0.283 \\
Stroke & 4 & 3 & 0.279 \\
Duración FA (años) & $4.9 \pm 2.1$ & $3.1 \pm 1.3$ & 0.306 \\
Tamaño Al (cm) & $6.1 \times 7.4 \times 6.2$ & $5.8 \times 7.2 \times 5.9$ & 0.223 \\
Trombo Al & 8 & 1 & 0.309 \\
FEV| & $53.5 \pm 6$ & $51.4 \pm 7$ & 0.421 \\
PSAP (mm Hg) & $50 \pm 16$ & $61 \pm 13$ & 0 \\
Antiarrítmicos $(\geq 2)$ & 36 & 14 & 0 \\
Síntomas FA & 36 & 14 & 0.308 \\
Diabetes & 5 & 3 & 0.256 \\
Hipertensión & 7 & 5 & \\
\hline Al: aurícula izquierda & & & \\
\hline
\end{tabular}

Al: aurícula izquierda; FEVI: fracción de eyección del ventrículo izquierdo; PSAP: presión sistólica de la arteria pulmonar; RF: radiofrecuencia bipolar intraoperatoria.

a El tamaño de la aurícula izquierda es obtenido en centímetros por ETT bidimensional como diámetros superoinferior $\times$ anteroposterior $\times$ transverso.

\section{Resultados}

Los resultados de ambos grupos se muestran en la tabla 2. Hubo 2 casos de mortalidad operatoria en el grupo 1 . Los tiempos de circulación extracorpórea y de pinzado aórtico fueron más largos en el grupo I $(175 \pm 22$ y $135 \pm 28 \mathrm{~min}$, respectivamente) que en el grupo ॥ (142 \pm 31 y $106 \pm 22 \mathrm{~min}$, respectivamente). Sin embargo, no hubo diferencia estadísticamente significativa $(p=0.399$ y $p=0.371$, respectivamente). Hubo 3 casos de reoperación por sangrado, 2 en el grupo 1 y uno en el grupo $॥(p=0.652)$.
Se observó una marcada tendencia hacia la colocación de bioprótesis mitral en el grupo I ( 28 casos) que en el grupo ॥ ( 2 casos), con un valor de $p<0.001$. Respecto al análisis del punto final, $33(92 \%)$ de los casos del grupo I estaban libres de FA a los 3 y 6 meses, en comparación con 7 (53\%) del grupo II. Más aún, el $77 \%$ de los pacientes del grupo I estaban libres de tratamiento con anticoagulantes orales al momento del seguimiento (a los 3 y 6 meses postoperatorios) contra el $21 \%$ del grupo II, con un valor de $\mathrm{p}<0.001, \mathrm{OR}=12.8$ (IC95\%: 2.8-57.4). En cuanto a la instalación de marcapasos definitivo, esta fue necesaria en 3 casos del grupo । (8.8\%) y en

Tabla 2 Resultados en ambos grupos de pacientes

\begin{tabular}{lllr}
\hline Variable & Grupo I & Grupo II & P \\
Tipo Cox-maze & III (corte-y-sutura) & IV (RF) & 0.313 \\
Mitral & 36 & 14 & 0.308 \\
Reparación mitral & 2 & 3 & $<0.001$ \\
Bioprótesis mitral & 28 & 2 & 0.297 \\
Prótesis mitral mecánica & 6 & 9 & 0.265 \\
Otros (adicional a mitral) & 5 & 2 & 0.399 \\
DCP (min) & $175 \pm 22$ & $142 \pm 31$ & 0.371 \\
Pinzado aórtico (min) & $135 \pm 28$ & $106 \pm 22$ & 0.462 \\
Sangrado postoperatorio (ml) & $555 \pm 218$ & $666 \pm 222$ & 0.652 \\
Reoperación por sangrado & 2 & 1 & 0.744 \\
Stroke & 0 & 1 & 0.283 \\
Estancia UCI & $4 \pm 2$ & $3 \pm 2$ & 0.268 \\
Estancia en piso & $8 \pm 6$ & $9 \pm 2$ & 0.923 \\
Mortalidad operatoria & 2 & 0 & $<(53 \%)$ \\
Libres de FA & $33(92 \%)$ & & $<0.001$ \\
\hline DCP: & & & \\
\hline
\end{tabular}

DCP: derivación cardiopulmonar; FA: fibrilación auricular; RF: radiofrecuencia bipolar intraoperatoria; UCl: unidad de cuidados intensivos. 
ningún caso del grupo $॥(p=0.30)$. Todos los pacientes que continuaban con FA después del tercer mes postoperatorio siguieron bajo un régimen medicamentoso a base de amiodarona (grupo ।, 3 [8.8\%]; grupo ॥, 7 [53\%]). El valor de $p<0.05$, $\mathrm{OR}=0.09$ (IC95\%: 0.02-0.44).

\section{Discusión}

Nuestros resultados confirman que la mejor opción de tratamiento quirúrgico para la FA sigue siendo el procedimiento de Cox-maze III estándar mediante corte-y-sutura, aun para pacientes pertenecientes al grupo de enfermedad valvular mitral reumática. La tasa de éxito para eliminación de la FA reportada por diversos autores empleando el procedimiento de Cox-maze III varía desde el 76 al $99 \%{ }^{13-15}$.

De acuerdo a la teoría de Allesie et al. ${ }^{16}$, se había pensado exclusivamente que la FA sucede en base a macrocircuitos de reentrada localizados en ambas aurículas. Consecuentemente, el procedimiento de maze o Cox-maze fue diseñado en base a varias incisiones quirúrgicas realizadas en las aurículas en un intento por evitar que las aurículas fibrilen ${ }^{17}$. Ahora sabemos que la génesis de la FA es en base a gatillos disparadores localizados habitualmente en las venas pulmonares $^{18}$. El mantenimiento de la misma está regulado por la existencia de uno o varios macrocircuitos de reentrada en una o en ambas aurículas ${ }^{19}$. El procedimiento de Cox-maze III completo debe seguir un patrón biauricular? ${ }^{9}$. Una parte clave en esta cirugía es que las incisiones quirúrgicas o líneas de quemadura no deben de ir separadas a una distancia superior a $5 \mathrm{~cm}^{17}$. Además, el procedimiento incluye el aislamiento de las venas pulmonares como parte fundamental de la técnica quirúrgica9 ${ }^{9}$. Es decir, la eficacia del procedimiento de Cox-maze III puede ser en gran medida explicada debido a que ataca a las 2 premisas anteriormente mencionadas. Por una parte aísla las venas pulmonares y con eso excluye el origen de la FA en dichas venas, y por otra, todas las incisiones van dirigidas a romper todos los macrocircuitos de reentrada en ambas aurículas. De hecho, nosotros obtuvimos el $93 \%$ de éxito respecto a la eliminación de la FA en los casos en los que se realizó Cox-maze III.

Desde su creación, el Cox-maze III ha sido severamente criticado debido a su alto grado de complejidad e invasividad. Es una cirugía que se realiza en circulación extracorpórea. Además, en una escala del 1 al 10 en grado de dificultad, esta operación ha sido evaluada en $9.5^{12}$. Las fuentes de energía surgen como alternativa a las incisiones quirúrgicas en base a la quemadura, en el entendido de que el fibrocito es la célula que conduce la electricidad en menor grado en toda la naturaleza. La radiofrecuencia y la criolesión son las más utilizadas ${ }^{20}$. La radiofrecuencia bipolar ha probado ser superior a la unipolar. Cuando se utiliza radiofrecuencia unipolar no se puede garantizar la transmuralidad ni la uniformidad de la quemadura lineal ${ }^{21}$. Todos nuestros casos del grupo ॥ fueron realizados utilizando radiofrecuencia bipolar. Existen detalles técnicos que pudieran explicar la superioridad del corte-y-sutura sobre la radiofrecuencia bipolar. Primero, en el corte-y-sutura no hay duda, se corta y se sutura. La división del tejido es inmediata, mientras que con la aplicación de radiofrecuencia el bloqueo conductivo de los macrocircuitos de reentrada está confinado a la fibrosis generada por la quemadura, la cual no siempre es inmediata. Segundo, el «planchado» para extender a la perfección la aurícula izquierda puede llegar a ser virtualmente imposible. Tercero, la línea de quemadura pudiera tener un efecto aislante del estímulo eléctrico casi inmediato. Sin embargo, se ha comprobado que casi el $40 \%$ del área de la quemadura estaría dada por edema, en lugar de necrosis tisular. Estas áreas de edema al resolverse y desaparecer pudieran generar hendiduras eléctricas en las mismas líneas de quemadura que pueden originar recidivas de la FA por reentradas en esos niveles. Nuestra casuística ha demostrado una amplia superioridad del corte-y-sutura sobre la aplicación de radiofrecuencia bipolar. La tasa de éxito eliminando la FA en un seguimiento a 6 meses fue del $92 \%$ para el grupo de corte-y-sutura contra el $53 \%$ para el de radiofrecuencia bipolar $(p<0.001)$.

Se observó una fuerte tendencia para la colocación de bioprótesis mitral cuando se realiza un procedimiento de Cox-maze III y cirugía valvular mitral $(\mathrm{p}<0.001 ; \mathrm{OR}=21$ [IC95\%: 3.87-113.88]). Creemos que la explicación para este fenómeno está fundamentada en 2 situaciones en particular. La primera es que dado el gran margen de seguridad que representa el Cox-maze III para eliminar la FA, es bastante congruente que la elección del cirujano sea hacia un procedimiento que evite la anticoagulación oral. La bioprótesis mitral y la reparación valvular mitral representan esa opción. Sin embargo, dada la baja incidencia de reparación valvular mitral que se presenta en el paciente reumático (todos los casos fueron reumáticos), es preferible instalar una bioprótesis en este rubro de pacientes. De hecho, en esta serie, 28 casos en el grupo । y 2 en el grupo ॥ $(p<0.001)$ fueron bioprótesis mitrales. Más aún, cuando combinamos ambos factores (Cox-maze III y bioprótesis mitral y/o reparación mitral) logramos evitar la anticoagulación a largo plazo en el $77 \%$ de los casos del grupo I y solo en el $21 \%$ del grupo II, con un valor de $\mathrm{p}<0.001$, OR = 12.8 (IC95\%: 2.8-57.4).

Tradicionalmente se ha mencionado que la alta incidencia de colocación de marcapasos definitivo en el Cox-maze III pudiera estar relacionada con una enfermedad del seno subyacente previa a la cirugía y enmascarada por la FA. De forma que cuando la FA se elimina, la enfermedad del seno enfermo es reconocida típicamente. Al revisar la técnica operatoria del Cox-maze III, no existe ninguna razón de peso para pensar en que alguna de las incisiones quirúrgicas pudiera lesionar el nodo sinusal o el auriculoventricular. Otra de las explicaciones es que pudiera tener relación con el gran edema que se forma como resultado de la tracción sobre el plano auricular ejercida en el momento de la cirugía. La exposición necesaria para llevar a cabo el Cox-maze III es mucho mayor que para el Cox-maze IV. En nuestra serie, se observó que la implantación de marcapasos definitivo fue necesaria en 3 casos del grupo । (8.8\%) y en ningún caso en el grupo $\|(p=0.30)$. Cabe mencionar que los 3 casos de implante de marcapasos definitivo fueron debido a bradicardia sinusal inferior a 50 latidos por minuto acompañados por sintomatología clínica de bajo gasto cardíaco.

Nuestro protocolo marca que después del tercer mes postoperatorio los medicamentos antiarrítmicos son suspendidos en ausencia de FA. Se ha tomado este tiempo debido a que es el tiempo que tardan las incisiones o líneas de quemadura en resolverse del edema. De hecho, el edema 
como tal puede ser una fuente de generación de microcircuitos de reentrada los cuales motivan la aparición de focos ectópicos disparadores de FA. Además, el edema tisular contribuye a hacer más corto el período refractario efectivo de las aurículas, lo cual es un factor predisponente para la aparición de FA. El período refractario efectivo se hace más pequeño y la masa crítica necesaria para la formación de un macrocircuito de reentrada también ${ }^{17}$. Nosotros tuvimos solo 3 casos $(8.8 \%)$ en el grupo I con necesidad de continuar el tratamiento con amiodarona más allá del tercer mes postoperatorio, y 7 casos $(53 \%)$ en el grupo ॥ $(p<0.05$, $\mathrm{OR}=0.09$ [IC95\%: 0.02-0.44]). Quizá la explicación para este fenómeno es que el edema es más intenso en los casos de Cox-maze IV debido a la quemadura por radiofrecuencia bipolar.

Finalmente, respecto a la no utilización de criolesión en los puntos clásicos descritos por Cox, como son el istmo mitral y el istmo cavo-tricuspídeo, existen reportes previos acerca de la no utilización de crioablación en el procedimiento de Cox-maze $1 I^{22}$. La premisa es que se tiene que dividir quirúrgicamente todo el espesor del tejido muscular hasta llegar a la grasa epicárdica, la cual no es conductora de electricidad. Nosotros hemos aplicado este concepto debido a que no contamos con crioablación en nuestra institución. No obstante, los resultados parecen ser bastante alentadores.

\section{Conclusiones}

Nuestros resultados son similares a los descritos previamente en la literatura. El procedimiento de Cox-maze III es superior a aquellos procedimientos que utilizan energías alternativas como substitutos de las incisiones quirúrgicas para eliminar la FA persistente de larga evolución. Especial énfasis debe hacerse en los excelentes resultados logrados aquí sobre un grupo de pacientes con enfermedad valvular mitral reumática, que es un substrato muy difícil de tratar debido a la gran fibrosis tisular que actúa en forma diferente a la FA no valvular en cuanto la génesis de esta arritmia.

\section{Responsabilidades éticas}

Protección de personas y animales. Los autores declaran que para esta investigación no se han realizado experimentos en seres humanos ni en animales.

Confidencialidad de los datos. Los autores declaran que han seguido los protocolos de su centro de trabajo sobre la publicación de datos de pacientes.

Derecho a la privacidad y consentimiento informado. Los autores han obtenido el consentimiento informado de los pacientes y/o sujetos referidos en el artículo. Este documento obra en poder del autor de correspondencia.

\section{Financiación}

Ninguna.

\section{Conflicto de intereses}

El autor declara no tener ningún conflicto de intereses.

\section{Bibliografía}

1. Colilla S, Crow A, Petkun W, et al. Estimates of current and future incidence and prevalence of atrial fibrillation in the U.S. adult population. Am J Cardiol. 2013;112: 1142-7.

2. Benjamin EJ, Wolf PA, D'Agostino RB, et al. Impact of atrial fibrillation on the risk of death: the Framingham Heart Study. Circulation. 1998;98:946-52.

3. Consultado 23 Jun 2015. Disponible en: http: / / www.stroke.org/ understand-stroke/preventing-stroke/afib-stroke-connection? pagename $=$ afib

4. Mozaffarian D, Benjamin EJ, Go AS, et al. Heart disease and stroke statistics -2015 update: A report from the American Heart Association. Circulation. 2015;131:e29-322.

5. Moukabary T, Naccarelli GV. Prevention of stroke in patients with atrial fibrillation. J Atrial Fibrillation. 2011;4:11-8.

6. Vázquez E, Martín A, Pousibet $\mathrm{H}$, et al. Utilización del tratamiento anticoagulante en los pacientes con fibrilación auricular no reumática. Rev Esp Cardiol. 2000;53:200-4.

7. Hart RG, Boop BS, Anderson DC. Oral anticoagulants and intracranial hemorrhage. Facts and hypotheses. Stroke. 1995;26:1471-7.

8. Hylek EM, Evans-Molina C, Shea C, et al. Major hemorrhage and tolerability of warfarin in the first year of therapy among elderly patients with atrial fibrillation. Circulation. 2007;115: 2689-96.

9. Cox JL, Jaquiss RD, Schuessler RB, et al. Modification of the maze procedure for atrial flutter and atrial fibrillation. II. Surgical technique of the maze III procedure. J Thorac Cardiovasc Surg. 1995;110:485-95.

10. Stulak JM, Suri RM, Burkhart HM, et al. Surgical ablation for atrial fibrillation for two decades: Are the results of new techniques equivalent to the Cox maze III procedure? J Thorac Cardiovasc Surg. 2014; 147:1478-87.

11. January CT, Wann LS, Alpert JS, et al. ACC/AHA Task Force Members 2014 AHA/ACC/HRS guideline for the management of patients with atrial fibrillation: Executive summary: a report of the American College of Cardiology/American Heart Association Task Force on practice guidelines and the Heart Rhythm Society. Circulation. 2014;130:2071-104.

12. Cox JL. Atrial fibrillation ॥ Rationale for surgical treatment. J Thorac Cardiovasc Surg. 2003;126:1822-8.

13. Stulak JM, Sundt TM 3rd, Dearani JA, et al. Ten-year experience with the Cox-maze procedure for atrial fibrillation: How do we define success? Ann Thorac Surg. 2007;83:1319-24.

14. Stulak JM, Suri RM, Burkhart HM, et al. Surgical ablation for atrial fibrillation for two decades: Are the results of new techniques equivalent to the Cox maze III procedure? J Thorac Cardiovasc Surg. 2014;147:1478-86.

15. Khargi K, Hutten BA, Lemke B, et al. Surgical treatment of atrial fibrillation; a systematic review. Eur J Cardiothorac Surg. 2005;27:258-65.

16. Allesie MA, Lammers WJEP, Bonke IM, et al. Intra-atrial re-entry as a mechanism for atrial flutter induced by acetilcholyne and rapid pacing in the dog. Circulation. 1984;70: 123-35.

17. Cox JL, Boineau JP, Schuessler RB, et al. The electrophysiologic basis, surgical development and clinical results of the maze procedure for atrial flutter and atrial fibrillation. Indian J Thorac Cardiovasc Surg. 1994;10:9-38. 
18. Haïssaguerre M, Jaïs $P$, Shah DC, et al. Spontaneous initiation of atrial fibrillation by ectopic beats originating in the pulmonary veins. N Engl J Med. 1998;339:659-66.

19. Cox JL. Atrial fibrillation I: A new classification system. J Thorac Cardiovasc Surg. 2003;126:1686-92.

20. García-Villarreal OA. eComment. Alternative energy sources in surgery for atrial fibrillation. Interact Cardiovasc Thorac Surg. 2012; 15:128. (una sola página).
21. Bugge E, Nicholson IA, Thomas SP. Comparison of bipolar and unipolar radiofrequency ablation in an in vivo experimental model. Eur J Cardiothorac Surg. 2005;28: 76-82.

22. Gregori F Jr, Cordeiro CO, Couto WJ, et al. Cox maze operation without cryoablation for the treatment of chronic atrial fibrillation. Ann Thorac Surg. 1995;60:361-3. 